\title{
Amélioration des propriétés physiques et chimiques du sol sous l'apport combiné des biodéchets et des engrais minéraux et influence sur le comportement du maïs (Zea mays L. variété Unilu)
}

Nyembo Kimuni Luciens', Useni Sikuzani Yannick¹, Chinawej Mbar Mukaz Dieudonné2, Kyabuntu llunga Dieudonné1, Kaboza Yambayamba ${ }^{3}$, Mpundu Mubemba Michel'1, Baboy Longanza Louis ${ }^{1}$

\author{
1 Faculté des Sciences Agronomiques, Université de Lubumbashi, Lubumbashi, BP 1825 RD Congo \\ 2 Département des sciences agronomiques et vétérinaires, section des sciences exactes, Institut Supérieur \\ Pédagogique de Lubumbashi, BP 1796 Lubumbashi, RD Congo \\ 3 Faculté des Sciences Agronomiques, Université de Kalémie, Kalémie RD Congo \\ Correspondance : luciensnyembokimuni@gmail.com; yannickuseni@gmail.com; michelmpundu@gmail.com
}

Original submitted in on 20 th January 2014 Published online at www.m.elewa.org on $28^{\text {th }}$ February 2014. http://dx.doi.org/10.4314/jab.v74i1.7

\section{RESUME}

Objectifs : Cette étude a été conduite au cours de la saison culturale 2012-2013 sur un ferralsol de la ferme Kasapa dans la région de Lubumbashi en vue d'évaluer les effets des doses combinées des fumiers des poules et des engrais minéraux sur le rendement de maïs (Zea mays Var Unilu) et certaines propriétés physiques et chimiques du sol.

Méthodologie et résultats : L'essai a été installé suivant un dispositif en split splot composé au total de 12 traitements et trois répétitions. Les traitements comprenaient 4 doses des fumiers des poules $(0,1,75$; $3,5$ et $7 \mathrm{t} / \mathrm{ha})$ et 3 doses des engrais minéraux $(0,300 \mathrm{~kg} \mathrm{NPK}+200 \mathrm{~kg}$ urée et $150 \mathrm{~kg} \mathrm{NPK}+100 \mathrm{~kg}$ urée). Des analyses physico-chimiques ont été réalisées sur des échantillons de sol et de fumiers de poules. La tendance générale de l'évolution des teneurs ( $\mathrm{N}$ et $\mathrm{K}$ ) était à la baisse par rapport aux valeurs initiales et les doses élevées des engrais minéraux et de biodéchets ont fourni des quantités élevées en $\mathrm{N}$ et $\mathrm{K}$. Par ailleurs, les biodéchets appliqués seuls ou en combinaison aux engrais minéraux ont amélioré la capacité de rétention d'eau. Le rendement a été largement affecté par l'apport des engrais minéraux que celui des biodéchets. La combinaison des fumiers de poules a permis d'accroitre le rendement en maïs grains $(6,9$ t.ha-1 pour $7 \mathrm{t}$ fumiers de poules combinés à $150 \mathrm{~kg} \mathrm{NPK}+100 \mathrm{~kg}$ urée) alors que le rendement le plus faible (2,9 t.ha-1) a été obtenu sur les parcelles témoins.

Conclusion et application de la recherche: L'étude a montré que les fumiers de poules combinés aux engrais minéraux ont permis d'accroitre le rendement du maïs dans les conditions de cet essai. Les fumiers de poules présentent un grand potentiel pour l'amélioration de la disponibilité des éléments nutritifs de sol et a pu fournir la quantité des nutriments nécessaires à la culture du maïs.

Mots clés : maïs, biodéchets, rendement, ferme Kasapa, engrais minéraux, fumiers de poules. 
ABSTRACT

\section{Combined effects of biowastes and mineral fertilizers on soil properties and maize yield in Lubumbashi, DR Congo}

Objectives: This study was conducted during 2012-2013 growing season on the Ferralsol Kasapa farm in Lubumbashi region to assess the effects of combined doses of manure and mineral fertilizers on the yield of maize (Zea mays Var UNILU) and some physico-chemical properties of soil.

Methodology and results: The trial was installed following a split splot device comprises in total 12 treatments and three repetitions. The treatments included four doses of manure from chickens $(0,1.75,3.5$ and 7 t.ha- $\left.^{-1}\right)$ and three doses of mineral fertilizer $(0,300 \mathrm{~kg} \mathrm{NPK}+200 \mathrm{~kg}$ urea by hectare and $150 \mathrm{~kg} \mathrm{NPK}$ $+100 \mathrm{~kg}$ urea by hectare). Physico-chemical analyzes were performed on samples of soil and biowastes. The general trend of the evolution of levels ( $\mathrm{N}$ and $\mathrm{K}$ ) was decreased compared to baseline and high doses of mineral fertilizers and organic waste provided high amounts of $\mathrm{N}$ and $\mathrm{K}$. Yield were significantly affected by the supply of minerals that of biowastes. The combination of chicken manure helped increase maize yield ( 6.9 t.ha $^{-1}$ of maize grain at 7 tha $^{-1}$ biowastes combined to $150 \mathrm{~kg} \mathrm{NPK}+100 \mathrm{~kg}$ urea), while the lowest yield $\left(2.9\right.$ t.ha- $\left.^{-1}\right)$ was obtained on the control plots.

Conclusion and research application: The study showed that biowastes combined with inorganic fertilizers increased maize yield in the conditions of this test. Biowaste have great potential for improving the availability of nutrients from soil and was able to provide the amount of nutrients needed for maize growing. KEYWORDS: maize, organic waste, yield, farm Kasapa, mineral fertilizers, manure from chickens.

\section{INTRODUCTION}

Les sols des pays d'Afrique subsaharienne ont un faible niveau de fertilité intrinsèque lié à des contraintes naturelles spécifiques à chaque zone agro écologique. Ces déficiences sont des facteurs déterminants de la production (Nyembo et al., 2012). Elles sont méconnues si bien que de faibles rendements perdurent. Les éléments nutritifs exportés par les récoltes, dans des sols déjà pauvres, ne sont pas remplacés de manière adéquate (Mulaji, 2011). Cette pratique, combinée à la pression foncière, a accentué la pauvreté des sols et favorisé la migration des agriculteurs à la recherche de nouvelles terres cultivables vers les aires protégées. Dès lors, la disponibilité des terres cultivables, le problème de leur dégradation et de leur reconstitution sont devenus une préoccupation majeure à l'échelle nationale (Kasongo et al., 2013). II apparaît alors urgent de développer des techniques de fertilisation de courte durée et plus facilement accessibles aux paysans. Pour pallier à ces baisses, il s'agit entre autre, de la fertilisation minérale par les engrais chimiques, de la fertilisation organique par l'utilisation de compost, d'engrais vert, de légumineuses fixatrices d'azote atmosphérique (Gala et al., 2011 ; Kaho et al., 2011 ; Nyembo et al., 2012; Useni et al., 2012 ; Kasongo et al., 2013). Ces différentes propositions ont une incidence financière que ne peut supporter le paysan qui pratique une agriculture de subsistance. Parallèlement, les villes offrent de quantités importantes de biodéchets renfermant entre 50 et $90 \%$ de matière organique, et pour lesquels la collecte et le traitement constituent un cout (Kaho et al., 2011) Une des voies prometteuses pour accroitre la production agricole en milieu paysan consiste à apporter aux sols différents types de matière organique et des engrais minéraux de sorte à accroître la disponibilité des éléments nutritifs du sol (Nyembo et al., 2013; Mulaji, 2011). En général, les systèmes durables à haut rendement ne sont obtenus qu'avec un niveau adéquat de matière organique et un niveau optimal de teneurs en éléments nutritifs du sol. Les études conduites en milieu naturel et en serre (laboratoire) ont montré que les ressources locales comme les déchets organiques et les roches naturelles, appliquées aux sols tropicaux pauvres et acides peuvent fournir les éléments nutritifs nécessaires pour l'alimentation et la croissance des plantes et par conséquent, accroître le rendement des plantes 
cultivées (N'Dayegamiye et al., 2004 ; N'Dayegamiye et al., 2005 ; Mulaji, 2011 ; Mpundu et al 2013). Ce travail, conduit à la ferme Kasapa au cours de la saison culturale 2012-2013, a été

\section{MILIEU, MATERIEL ET METHODES}

Description du site d'étude : L'essai a été conduit au cours de la saison culturale 2012-2013 à la ferme Kasapa, au Nord-ouest de la ville de Lubumbashi en RDC (Figure 1). Conduite à la ferme Kasapa, l'expérimentation a été localisée dans un carré formé par les latitudes $11^{\circ} 35^{\prime} 08,5^{\prime \prime}$ et $11^{\circ} 35^{\prime} 07,2^{\prime \prime} \mathrm{S}$ et les longitudes $27^{\circ} 24^{\prime} 46,3^{\prime \prime}$ et $27^{\circ} 24$ ' $45,9^{\prime \prime}$ E à $1258 \mathrm{~m}$ d'élévation. La région de Lubumbashi est caractérisée par un climat du type Cw6 de la classification de initié dans la perspective de l'augmentation de la production du maïs par la valorisation des déchets organiques (fumier des poules).

Koppën (FAO, 2005). Elle est caractérisée par une période de croissance normale d'une durée moyenne de 182 jours en moyenne elle est constituée d'une période humide d'environ 150 jours. Cette période de croissance commence à la seconde moitié d'octobre pour s'arrêter vers la mi-avril tandis que la période humide va de la première moitié de novembre jusqu'à la première décade d'avril (Kasongo et al., 2013).

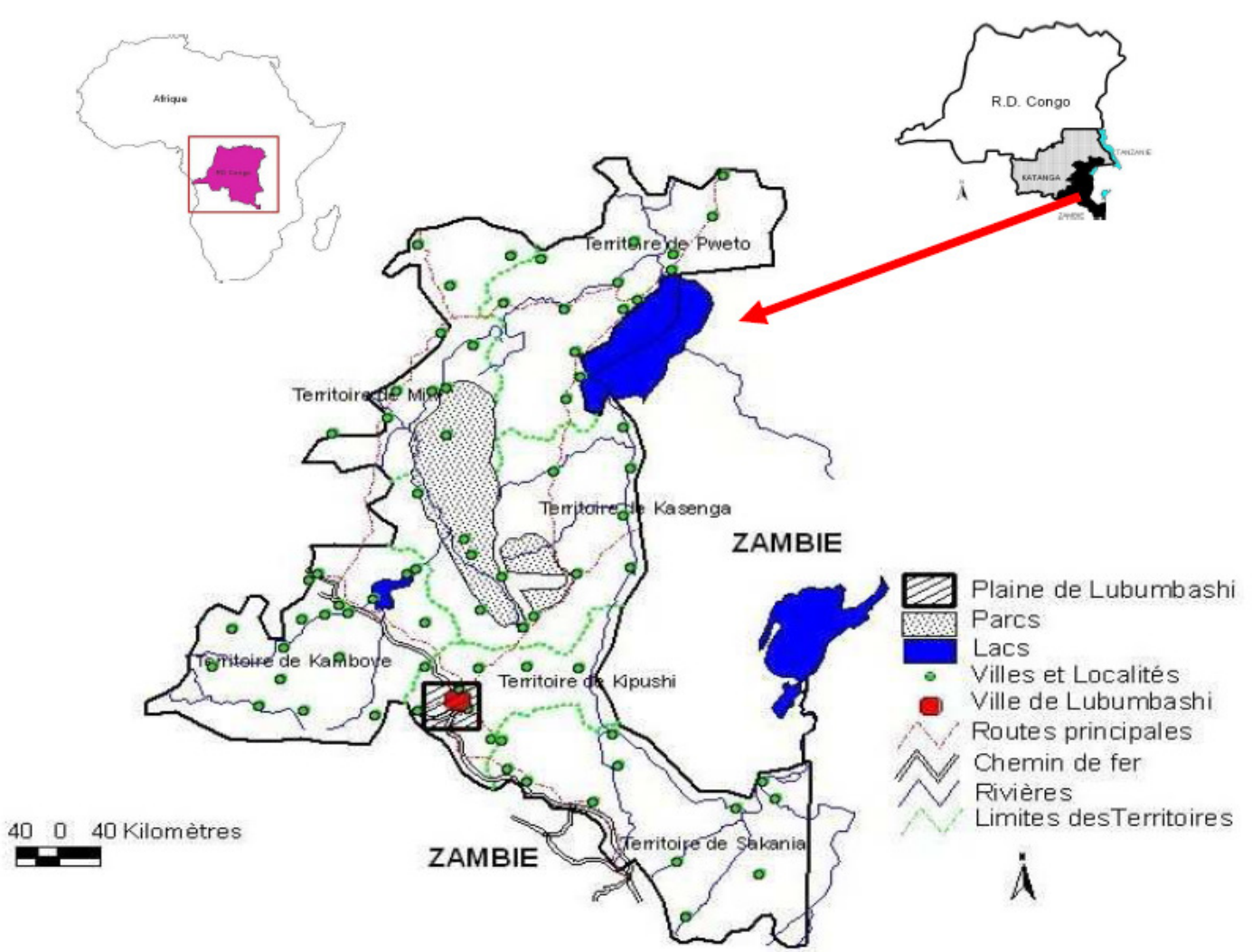

Figure 1 : Localisation de la ville de Lubumbashi dans le district du Haut-Katanga, province du Katanga, la RD Congo et le continent Africain (Munyemba, 2010)

Jadis, l'essentiel de la végétation était constitué par les formations végétales allant de la forêt claire dite "Miombo » aux galeries forestières (Mushitu), en passant par la forêt dense sèche appelée «Muhulu» (Munyemba et al., 2008). Actuellement l'occupation du sol autour de Lubumbashi a profondément changé et la savane correspond à la dégradation de la forêt claire et les stades intermédiaires (savanes arborées et arbustives) s'observent en de très nombreux endroits (Munyemba, 2010; Cabala, 2012). La flore de recolonisation du site expérimental était dominée par Imperata cylindrica, Cynodon dactylon et Tithonia 
diversifolia qui occupent le terrain à des degrés de recouvrement variés. La couverture pédologique est du type ferralitique avec un $\mathrm{pH}$ à l'eau oscillant autour de 5 ,2. En général, les sols de Lubumbashi sont du type ferralitique, profondément altérés qui se caractérisent notamment par l'abondance de sesquioxydes de fer et d'alumine et de matériaux argileux à faible capacité d'échange (kaolinite). La CEC rapportée à 100\% d'argile ne peut théoriquement dépasser $16 \mathrm{Cmole.} \mathrm{kg}$ ${ }^{1}$ (Kasongo et al., 2013).

Matériels d'étude : La variété Unilu a été utilisée comme matériel biologique, plus cultivée dans la région, à côté des variétés Katanga et Babungo. Cette variété est plus préférée en raison de sa résistance aux maladies (helminthosporiose, cercosporiose et striure), aux ravageurs, et son potentiel élevé de rendement $(7$ à 8,5 t.ha $^{-1}$ à une densité de 53333 plants ha- ${ }^{-1}$ ). En plus, elle est mieux indiquée pour la faible taille des plantes qui la rend moins sensible à la verse (Nyembo et al., 2012; Useni et al., 2012). Les fumiers des poules utilisés dans le cadre de cette étude provenaient de la ferme avicole DAIPN, située au quartier Kilobelobe. Le choix de ce site a été motivé par l'élevage intensif qui y est pratiqué et par rapport au faible coût des fumiers $(0,11 \$$ par $\mathrm{kg}$ soit 9.96 en FC). L'analyse chimique de ces fumiers au laboratoire de Chimie, à la Faculté des Sciences (Université de Lubumbashi) a donné les résultats suivants: $\mathrm{N}$ $(2,1532 \%)$ et $\mathrm{K}(0,0031 \%)$. L'engrais composé NPK (10-20-10) fabriqué par Omnia, utilisé comme engrais de fond et l'urée $(46 \% \mathrm{~N})$, comme engrais de couverture, ont été apportés à des quantités différentes en fonction des traitements. Le coût d'acquisition des engrais minéraux étaient respectivement de $1 \$$ soit $900 \mathrm{Fc}$ et $1,06 \$$ soit $954 \mathrm{Fc}$ le kilogramme.

Méthodologie: L'essai a été installé suivant un dispositif en split splot composé au total de 12 traitements et trois répétitions. Les traitements comprenaient 4 doses des fumiers des poules $(0,1,75$; 3,5 et 7 t/ha) et 3 doses des engrais minéraux ( 0 , $300 \mathrm{~kg} \mathrm{NPK}+200 \mathrm{~kg}$ urée comme fumure minérale vulgarisée et $150 \mathrm{~kg} \mathrm{NPK}+100 \mathrm{~kg}$ urée comme la fumure minérale vulgarisée réduite de moitié). Le choix de notre terrain d'expérimentation a porté sur un ancien terrain où le défrichement n'a pas été effectué et la

\section{RESULTATS}

Effets des apports combinés des biodéchets et des engrais minéraux sur les propriétés physiques et chimiques du sol: Les résultats obtenus sur la préparation du terrain était limitée au labour suivi d'un hersage; les deux opérations étant mécanisées. La délimitation des parcelles a été effectuée à l'aide d'un décamètre et des piquets. Les grains de maïs ont été semés en lignes à une densité de 53333 plantes par hectare ; ce qui correspond aux écartements de $0,75 \mathrm{~m}$ $x 0,25 \mathrm{~m}$. Compte tenu des mauvaises herbes qui envahissaient le champ expérimental, le premier sarclage est intervenu 21 jours après semis. Le deuxième sarclage a été combiné avec le buttage et ces deux opérations sont intervenues 45 jours après semis. A la récolte, les épis des maïs ont été récoltés sur les deux lignes du milieu et le rendement a été ajustés au taux d'humidité de $14 \%$. Avant et après installation de l'essai, les échantillons des sols $(100 \mathrm{~g}$ environ par traitement) ont été prélevés et les opérations de préparation ont consisté au séchage à l'air libre, broyage à l'aide d'un mortier en porcelaine et d'un pilon approprié; et tamisage sur un tamis de $2 \mathrm{~mm}$ pour les analyses chimiques de routine pour l'estimation de la fertilité. Le pH eau, les teneurs en $\mathrm{N}$ minéral et $\mathrm{K}$ total ont été déterminés au laboratoire de la faculté des sciences, alors que la capacité de rétention en eau (CRC) ont été déterminés au laboratoire de la faculté des Sciences agronomiques de I'Université de Lubumbashi. La CRC du sol correspond à la teneur moyenne maximale en eau que peut retenir un sol contenu dans un pot de culture après saturation complète et ressuyage libre. La CRC a été déterminée en plongeant les boites trouées contenant les échantillons dans une bassine d'eau en veillant à ce que le niveau d'eau dans la bassine ne dépasse pas le niveau du sol dans les boites. L'eau monte par capillarité dans les boites. Après 24 heures, les boites sont retirées de la bassine et laissées s'égoutter pendant encore 24 heures. Le seuil en dessous duquel l'eau contenue dans le sol ne peut pas être drainée ou l'humidité résiduelle représente la différence de poids après séchage de l'échantillon à $105^{\circ} \mathrm{C}$ pendant 24 heures (Mulaji, 2011). Les résultats bruts ont été soumis à l'analyse de la variance (ANOVA). II s'agit d'une ANOVA multifactorielle (effets des doses de fumiers des poules, effets des doses d'engrais minéraux et leur interaction) avec test post hoc de TUKEY.

dynamique des propriétés chimiques des sols sont présentés dans le tableau 1 
Tableau 1. Effet des différents traitements de fumiers des poules combinés aux engrais inorganiques sur la teneur en $\mathrm{N}$ et $\mathrm{K}$ du sol.

\begin{tabular}{ccc}
\hline & \multicolumn{3}{c}{ Teneurs en éléments minéraux $\mathbf{( 0 - 2 0} \mathbf{c m}$ ) avant et à la fin de l'essai } \\
\hline Initial (Avant l'essai) & $\mathbf{N} \%$ & $\mathbf{K} \%$ \\
& 0,6916 & 0,0026 \\
Traitement & & \\
T0 & 0,26 & 0,0007 \\
T1 & 1,6 & 6,908 \\
T2 & 4,28 & 2,565 \\
T3 & 3,0 & 2,754 \\
T4 & 2,47 & 2,366 \\
T5 & 1,9 & 3,947 \\
T6 & 4,2 & 14,21 \\
T7 & 3,9 & 15,58 \\
T8 & 6,8 & 10,23 \\
T9 & 2,55 & 11,171 \\
T10 & 4,65 & 4,214 \\
T11 & 7,22 & 2,581 \\
\hline
\end{tabular}

T0 (sans fertilisants) ; T1 (1,75 tonnes fumiers poules sans engrais minéral); T2 (3,5 tonnes fumiers poules sans engrais minéral) ; T3 (7 tonnes fumiers poules sans engrais minéral) ; T4 (150 kg NPK+100 kg urée) ; T5 (1,75 tonne de fumier des poules, $150 \mathrm{~kg} \mathrm{NPK}+100 \mathrm{~kg}$ urée) ; T6 (3,5 tonnes de fumiers de poules, $150 \mathrm{~kg} \mathrm{NPK+100} \mathrm{kg} \mathrm{urée)} \mathrm{;} \mathrm{T7} \mathrm{(7} \mathrm{tonnes} \mathrm{de} \mathrm{fumiers} \mathrm{de}$ poules, $150 \mathrm{~kg} \mathrm{NPK}+100 \mathrm{~kg}$ urée) ; T8 (300 kg NPK $+200 \mathrm{~kg}$ urée, sans fumiers de poules); T9 (1,75 tonnes de fumiers de poules, $300 \mathrm{~kg} \mathrm{NPK}+200 \mathrm{~kg}$ urée) ; T10 (3,5 tonnes de fumiers de poules, $300 \mathrm{~kg}$ NPK $+200 \mathrm{~kg}$ urée); T11 (7 tonnes de fumiers de poules, $300 \mathrm{~kg} \mathrm{NPK}+200 \mathrm{~kg}$ urée)

En fin de l'essai, les teneurs en $\mathrm{N}$ et $\mathrm{K}$ étaient très faibles par rapport aux traitements ayant reçu les fertilisants. En ce qui concerne les doses de biodéchets, les résultats montrent que la teneur en $\mathrm{N}$ était plus élevée sur les traitements à dose moyenne des biodéchets alors que celle du K l'est sur le traitement à dose élevée de biodéchets. En revanche, les teneurs en $\mathrm{N}$ et $\mathrm{K}$ obtenus sur les traitements avec engrais minéraux sont proportionnelles aux doses appliquées. Les teneurs ( $\mathrm{N}$ et $\mathrm{K}$ ) étaient supérieures par rapport aux valeurs initiales. Par ailleurs, les valeurs ont été plus élevées dans les parcelles ayant reçues les fumiers des poules combinés aux fumures minérales vulgarisées sur deux (T7 et T6).Les résultats sur les effets des doses croissantes de fumiers de poules, des engrais minéraux et de leur combinaison sur la CRC sont présentés sur les figures 2, 3 et 4. Les résultats de l'analyse de la variance montrent que la CRC n'a pas été influencée par les doses de fumiers de poules $(P=0,138)$, les doses des engrais minéraux $(P=0,735)$ et leur interaction $(P=0,667)$. 


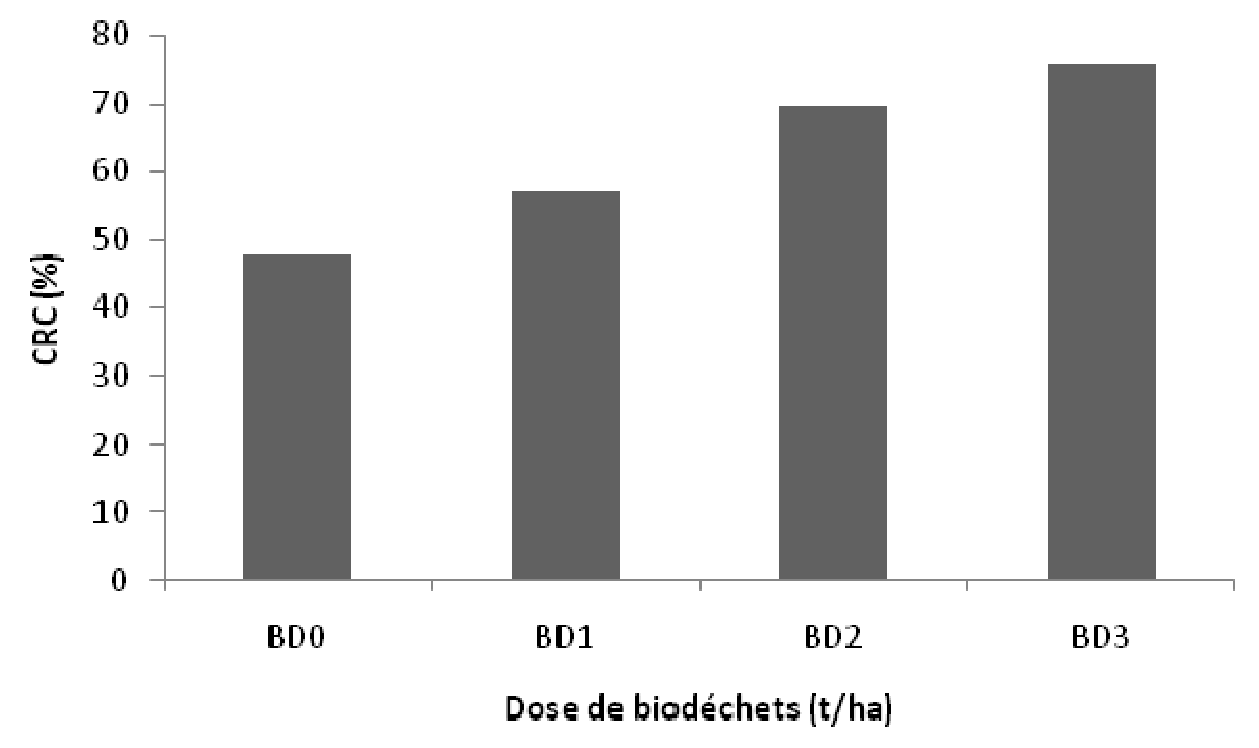

Figure 2 : Effets des doses croissantes de fumiers de poules sur la capacité de rétention d'eau. BDO $0=$ sans fumiers de poules ; $B D 1=1,75$ t.ha-1 de fumiers de poules ; $B D 2=3,5$ tha-1 de fumiers de poules ; $B D 3=7,5$ t.ha-1 de fumiers de poules

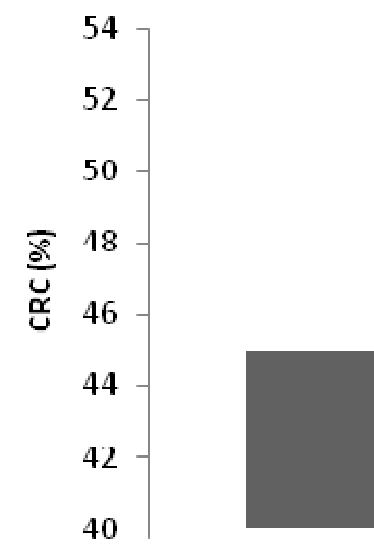

EIM0

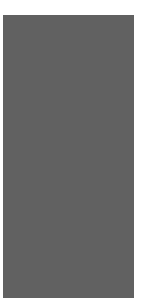

HMV

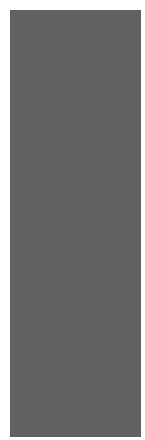

HMV/2

Doses des engrais minéraux

Figure 3 : Effets des doses croissantes des engrais minéraux sur la capacité de rétention d'eau. EM $0=$ sans engrais

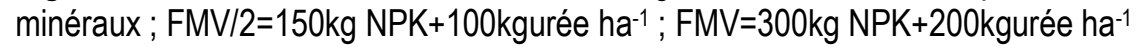




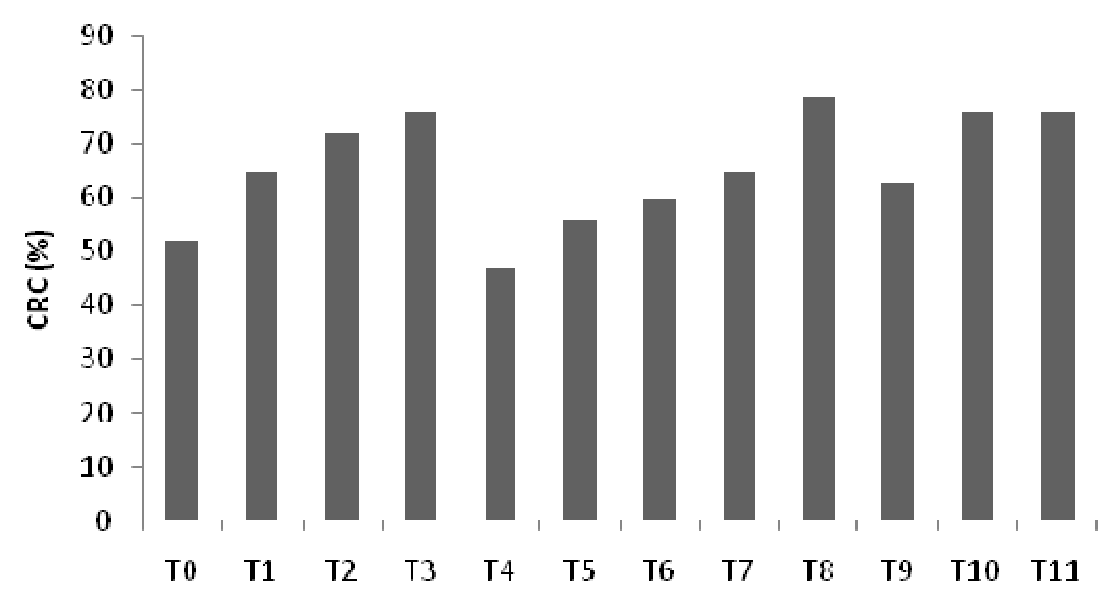

Apports combinés de biodéchets êt dés engrais minéraux

Figure 4 : Effets combinés des doses croissantes des engrais minéraux et des fumiers de poules sur la capacité de rétention d'eau.

T0 (sans fertilisants) ; T1 (1,75 tonnes fumiers poules sans engrais minéral) ; T2 (3,5 tonnes fumiers poules sans engrais minéral) ; T3 (7 tonnes fumiers poules sans engrais minéral) ; T4 (150 kg NPK+100 kg urée) ; T5 (1,75 tonne de fumier des

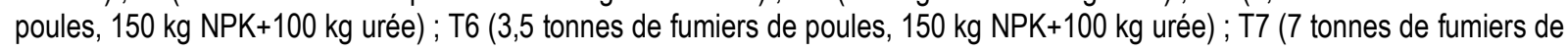
poules, $150 \mathrm{~kg} \mathrm{NPK}+100 \mathrm{~kg}$ urée) ; T8 (300 kg NPK $+200 \mathrm{~kg}$ urée, sans fumiers de poules); T9 (1,75 tonnes de fumiers de poules, $300 \mathrm{~kg} \mathrm{NPK}+200 \mathrm{~kg}$ urée) ; T10 (3,5 tonnes de fumiers de poules, $300 \mathrm{~kg}$ NPK $+200 \mathrm{~kg}$ urée); T11 (7 tonnes de fumiers de poules, $300 \mathrm{~kg} \mathrm{NPK}+200 \mathrm{~kg}$ urée)

Réponse de la culture de maïs: La variation du rendement de maïs en fonction des doses des engrais minéraux et de fumier des poules est résumée sur les figures 5,6 et 7 . L'analyse de variance indique l'existence d'un effet d'engrais minéraux sur l'ensemble des paramètres de rendement. La comparaison des moyennes de ces paramètres montre une amélioration de rendement de maïs $(p<0,05)$
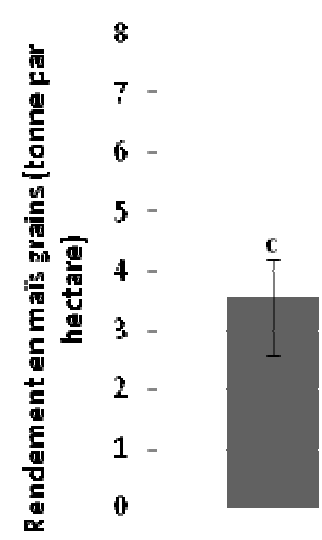

KIIII

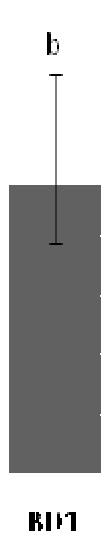

Sostse de biodśches:e grâce à l'apport des doses d'engrais minéraux. En effet, les rendements de 5,9 tha-1 ont été obtenus sur les parcelles fertilisées à la forte dose contre 3,6 tha-1 sur les parcelles non fertilisées. Par contre, les doses des fumiers de poules et les interactions engrais*fumiers de poules ont induit des effets similaires sur l'ensemble des paramètres de rendement.

Figure 5. Rendement du maïs en fonction des doses de biodéchets. $B D 0=$ sans fumiers de poules ; $B D 1=1,75$ tha $^{-1}$ de fumiers de poules ; $B D 2=3,5$ t.ha-1 de fumiers de poules ; $B D 3=7,5$ tha-1 de fumiers de poules 


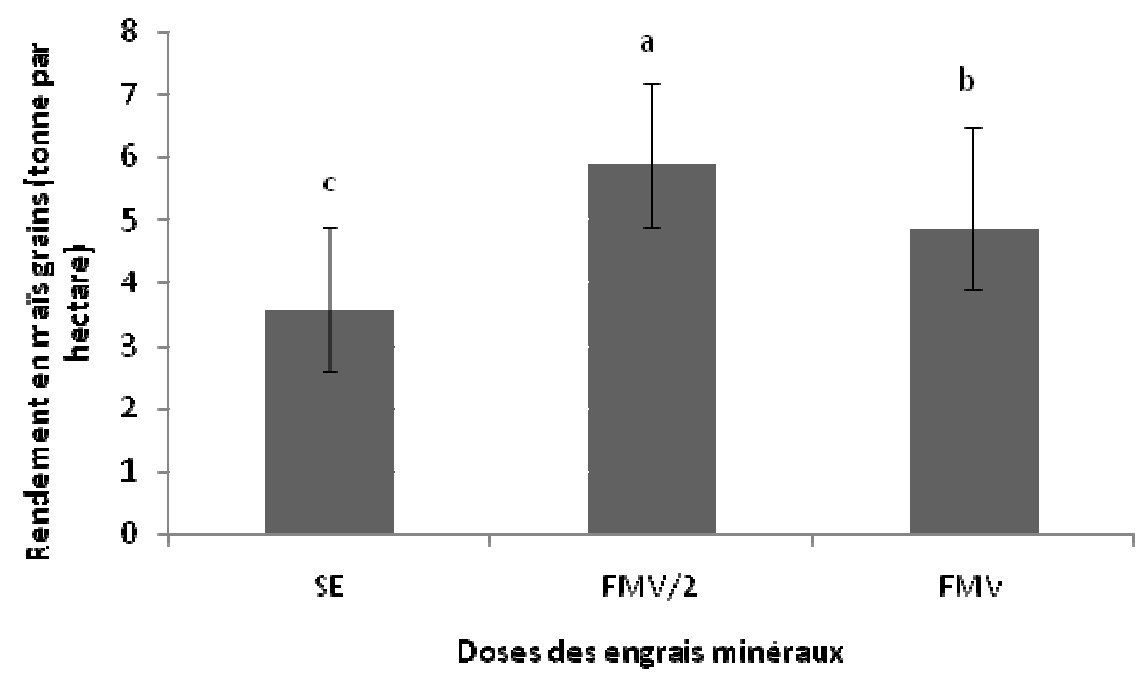

Figure 6 : Rendement du maïs en fonction des engrais minéraux. SE $0=$ sans engrais minéraux; $F M V / 2=150 \mathrm{~kg}$ NPK+100kgurée ha-1 ; FMV=300kg NPK+200kgurée ha-1

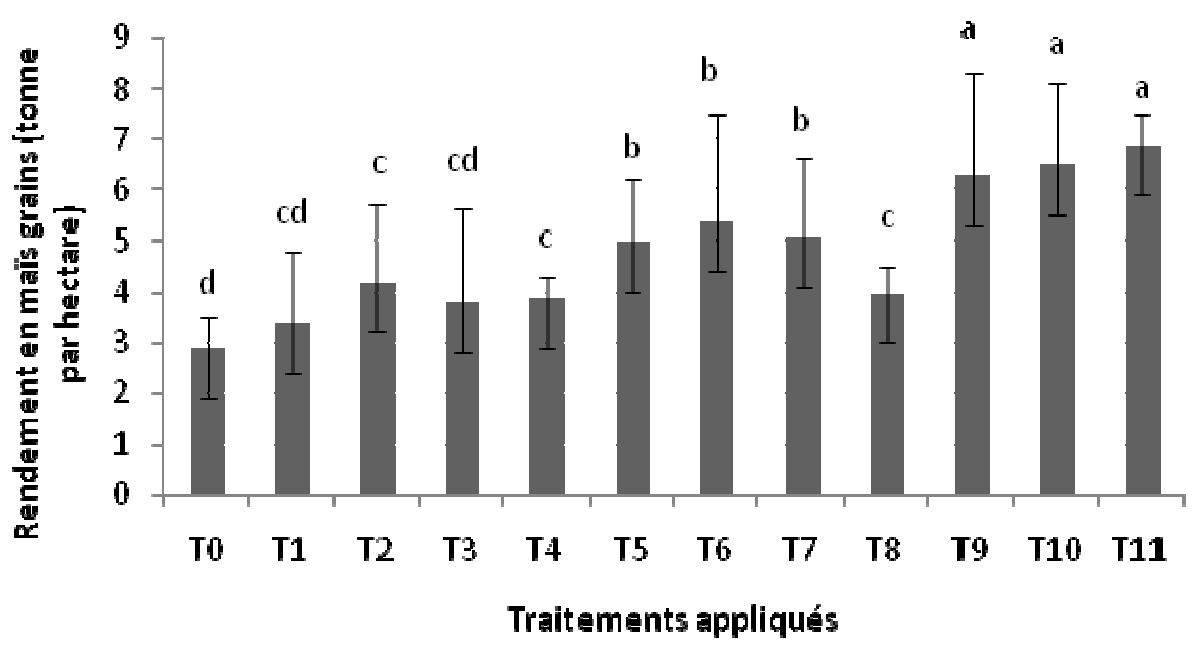

Figure 7. Effets combinés des doses croissantes des engrais minéraux et des fumiers de poules sur le rendement du maïs.

T0 (sans fertilisants) ; T1 (1,75 tonnes fumiers poules sans engrais minéral); T2 (3,5 tonnes fumiers poules sans engrais minéral) ;T3 (7 tonnes fumiers poules sans engrais minéral) ; T4 (150 kg NPK+100 kg urée) ; T5 (1,75 tonne de fumier des

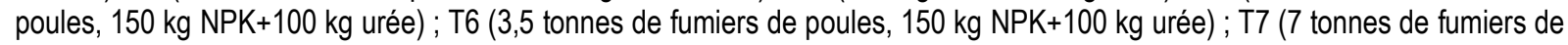
poules, $150 \mathrm{~kg} \mathrm{NPK}+100 \mathrm{~kg}$ urée) ; T8 (300 kg NPK $+200 \mathrm{~kg}$ urée, sans fumiers de poules); T9 (1,75 tonnes de fumiers de poules, $300 \mathrm{~kg}$ NPK $+200 \mathrm{~kg}$ urée) ; T10 (3,5 tonnes de fumiers de poules, $300 \mathrm{~kg}$ NPK $+200 \mathrm{~kg}$ urée); T11 (7 tonnes de fumiers de poules, $300 \mathrm{~kg} \mathrm{NPK}+200 \mathrm{~kg}$ urée)

\section{DISCUSSION DES RESULTATS}

En observant le tableau 1, l'analyse du laboratoire révèle que les teneurs ( $\mathrm{N}$ et $\mathrm{K}$ ) étaient supérieures par rapport aux valeurs initiales. Ceci suggère que la synchronisation de la libération des éléments nutritifs par les fumiers des poules combinés aux engrais inorganiques pendant leur décomposition et leur assimilation par les plantes n'ont pas coïncidée avec le moment où l'assimilation des nutriments est au pic. En effet, Kaho et al. (2011) ont montré que le taux de décomposition de la matière organique et 
l'augmentation des rendements était étroitement lié à la synchronisation entre la libération des nutriments et leur assimilation par la plante. Néanmoins, des teneurs élevées en $\mathrm{N}$ et $\mathrm{K}$ obtenus sur les parcelles fertilisées aux biodéchets confirment que la matière organique améliore les propriétés chimiques du sol, les rendant favorable à la croissance des cultures et est une source d'éléments nutritifs pour les cultures (Mulaji, 2011). Les résultats obtenus lors d'analyse du sol au laboratoire avant et à la fin de l'essai ont montré la faible biodisponibilité pour l'azote et le potassium sur les traitements témoins. Ceci serait dû aux caractéristiques des sols acides : pH acide et toxicité Al. En plus, sur les parcelles témoins, l'absence d'apports organiques s'accompagne d'une perte en matières organiques et en nutriments, d'une acidification des sols, d'une réduction de la biomasse et de l'activité microbienne, d'une insolubilisation du potassium qui ensemble contribuent à la baisse sensible des rendements des cultures (Deblay, 2006). Les apports de matières organiques améliorent le $\mathrm{pH}$ du sol aussi bien en sol acide par son pouvoir tampon et les apports des bases ( $\mathrm{Ca}, \mathrm{Mg}, \mathrm{K}, \mathrm{Na}$ ) qu'en sol basique par l'augmentation de la capacité d'échange cationique (CEC) au niveau des complexes argilo-humiques. Ces complexes augmentent le pouvoir tampon du sol et sa capacité à résister aux variations de $\mathrm{pH}$ (Charland et al., 2001). Par rapport aux engrais chimiques, ils apportent, en plus des macroéléments, un complément d'oligoéléments très favorables à la végétation (Gobat et al., 1998). Les études conduites en milieu naturel et en serre (laboratoire) ont montré que les ressources locales comme les déchets organiques et les roches naturelles, appliquées aux sols tropicaux pauvres et acides peuvent fournir les éléments nutritifs nécessaires pour l'alimentation et la croissance des plantes et par conséquent, accroître le rendement des plantes cultivées (N'Dienor, 2006; N'Dayegamiye et Drapeau, 2009; Kochi et al., 2010 ; Kaboré et al., 2011). L'apport des fumiers des poules a considérablement influencé tous les paramètres de rendement. Le poids de grains par épi et le rendement en maïs grain ont été sensiblement affecté par l'apport des différentes doses des fumiers des poules. Les amendements ont permis d'améliorer les rendements en maïs grain par rapport au témoin non fertilisé. L'apport de 7 t.ha $^{-1}$ combiné aux FMV/2 a amélioré de façon significative le rendement en maïs grain de 6,9 t.ha-1 $^{-1}$ par rapport aux rendements obtenus sur le sol témoin non amendé (2,9 t.ha-1). Par ailleurs, l'augmentation du rendement était directement proportionnelle aux doses de fumiers des poules appliquées. Ceci serait dû à la forte proportion d'éléments nutritifs libérés par la décomposition des fumiers des poules. L'augmentation de rendement serait due à l'amélioration des propriétés du sol (capacité de rétention d'eau) et à la libération des éléments nutritifs. Dans le même ordre d'idées, l'augmentation de rendement dû à l'apport des amendements organiques est attribuable au changement favorable des conditions du sol, entrainant un bon développement des racines et une bonne assimilation des éléments nutritifs libérés par la matière organique elle-même ou en retenant les éléments nutritifs libérés par les engrais, tel que démontré par Tejada et al. (2006) et Maftoun et al. (2004). Les résultats indiquent que les fumiers des poules apportés seuls n'ont pas permis d'obtenir des rendements élevés en maïs-grain, probablement à cause de la faible disponibilité de leurs éléments nutritifs, particulièrement l'azote. En effet, l'azote des fumiers des poules serait principalement sous forme organique et sa minéralisation en période de végétation n'a probablement pas été suffisante pour combler les besoins en $\mathrm{N}$ du maïs-grain. T0 a obtenu le rendement le plus bas $\left(2,9\right.$ t.ha- $\left.^{-1}\right)$. La faible production des sols témoins peut être attribuée aux facteurs caractéristiques des sols acides : pH acide, toxicité Al et $\mathrm{Mg}$, déficiences en nutriments $(\mathrm{Ca}, \mathrm{Mg}, \mathrm{P}, \mathrm{K}, \mathrm{B}$ et $\mathrm{Zn}$ ) et une réduction de la biomasse et de l'activité microbienne (Deblay, 2006; Nyembo et al., 2012). Dans les conditions de la région de Lubumbashi, Useni et al. (2013) ont obtenus des résultats similaires sur la culture de maïs avec utilisation de déchets humains recyclés. Les moyennes de rendement obtenues avec les doses sur les parcelles traitées aux engrais et à la forte dose des fumiers de poules $\left(7\right.$ t.ha $\left.^{-1}\right)$ sont similaires, mais supérieures à celles obtenues avec la dose FMV/2 (6,9 tha-1 tonnes) et le témoin (2,9 t.ha-1). Ceci montre que, comparativement au traitement à forte dose d'urée $(200 \mathrm{~kg})$, c'est plus le phosphore qui est l'élément limitant du rendement. L'apport des engrais chimiques a accru de manière significative le rendement du maïs comparativement aux parcelles témoins. En effet, le rendement obtenu sur les parcelles non fertilisées est 2 fois moins que ceux obtenus sur les parcelles fertilisées à la FMV. La faible production des sols témoins peut être attribuée aux facteurs caractéristiques des sols acides : pH acide, toxicité $\mathrm{Al}$ et $\mathrm{Mg}$, déficiences en nutriments (Mulaji, 2011 ; Kasongo et al., 2013). Les effets bénéfiques de la fertilisation chimique au moyen de l'agriculture ont 
été prouvés par de nombreuses recherches (Gala et al., 2011; Nyembo et al., 2012) Le rendement significativement plus élevé sur les parcelles fertilisées avec la dose de $300 \mathrm{NPK}+200 \mathrm{Kg}$ Urée à l'hectare pourrait être due à la libération des éléments importants : $280 \mathrm{Kg} \mathrm{N}, 100 \mathrm{Kg} \mathrm{P}$ et $50 \mathrm{Kg}$ de $\mathrm{K}$ à l'hectare qui sont en grande quantité. Pypers (2010) ont trouvé que les engrais augmentent 40 à $100 \%$ de rendement de culture dans les sols Kalongo et Civu, dans le Sud Kivu, en République Démocratique du Congo. Les résultats obtenus montrent que l'ajout des fumiers de poules aux sols fertilisés aux engrais minéraux a permis d'améliorer leurs effets tant sur les paramètres végétatifs que les paramètres de rendement. En effet, des rendements variant de 4,9 t.ha- ${ }^{-1}$ à 5,9 t.ha $^{-1}$ sont obtenus lorsque les fumiers des poules sont appliqués seuls contre 5 tha $^{-1}$ à 6,5 t.ha- $^{-1}$ si les engrais minéraux sont appliqués seuls. En dépit de leur faible et variable teneur en éléments nutritifs, la matière organique joue un rôle important dans la

\section{CONCLUSION}

La présente étude a été initiée en vue d'évaluer les effets des doses combinés des fumiers et des engrais minéraux sur le rendement de maïs (Zea mays Var Unilu) et certaines propriétés physico-chimiques d'un ferrasol de la ferme Kasapa. L'essai a été installé suivant un dispositif en split splot composé au total de 12 traitements et trois répétitions. Les traitements comprenaient 3 doses des engrais minéraux et 4 doses des fumiers des poules appliqués seuls et en combinaison.

Les résultats obtenus ont montré que :

- $\quad$ L'apport des biodéchets appliqués seuls ou en combinaison aux engrais minéraux améliorent la capacité de rétention d'eau ; des capacités de rétention d'eau similaire étant observés entre les traitements à engrais minéraux et le témoin. En outre, des stocks en éléments nutritifs n'ont été obtenus que sur les parcelles fertilisées.

- $\quad$ Les fumiers de poules appliqués seuls ou combinés aux engrais minéraux ont induit des différences significatives au niveau des paramètres du rendement.

\section{REFERENCES BIBLIOGRAPHIQUES}

Cabala KS, 2012. Dynamique spatiotemporelle de la forêt claire dans la plaine de Lubumbashi. Diplôme d'Etude Approfondie en Gestion des Ressources Naturelles et de Biodiversité de la gestion d'une meilleure fertilité du sol et d'en améliorer la structure (FAO et IFA, 2003). La combinaison d'engrais organique et minéraux crée les meilleurs conditions de production car la matière organique améliore les propriétés du sol alors que les engrais minéraux apportent aux plantes les éléments nutritifs qui leurs sont nécessaires et d'accroitre l'efficacité agronomique (Sanginga et Woomer, 2009). D'autres raisons justifient cette combinaison dont la déficience en éléments essentiels aux cultures des engrais minéraux communs alors que les ressources organiques les contiennent (Sanginga et Woomer, 2009). Seule la matière organique ne suffit pas souvent car elle n'est pas disponible en grande quantité pour assurer le niveau de production escompté pour les agriculteurs (Vanlauwe et al., 2006). Plusieurs études dont celles de Kaho et al (2011) et Useni et al (2012) ont montré que les rendements du maïs augmentent lorsque les engrais minéraux sont associés aux ressources organiques.

- Les traitements fertilisés aux doses des fumiers de poules ( 7 t.ha-1 $\left.^{-1}\right)$ associées à celles des engrais minéraux (300kg NPK et $200 \mathrm{~kg}$ d'urée) augmentent significativement le rendement du maïs par rapport aux parcelles fertilisées uniquement aux doses des engrais minéraux et aux témoins non fertilisés.

Toutefois, au regard des résultats obtenus, la dose de 1,75 t.ha $^{-1}$ des fumiers de poules est à recommander, connaissant le faible niveau de revenu des paysans de la zone d'étude.Les résultats de cette étude ont montré les fumiers de poules peuvent améliorer la fertilité du sol et augmenter de façon significative les rendements de culture. Dans les conditions de cet essai, les fumiers de poules présentent un grand potentiel pour l'amélioration de la disponibilité des éléments nutritifs de sol et peuvent fournir les quantités des nutriments nécessaires à la culture du maïs sans apport d'engrais inorganiques; ce qui est capital pour les petits agriculteurs de la zone d'étude. D'autres études sont cependant, nécessaires pour quantifier la durabilité de cet effet.

forêt claire, Université de Lubumbashi, Faculté des sciences agronomiques, $55 p$

Charland M, Cantin S, St Pierre MA, Côté L, 2001. Recherche sur les avantages à utiliser le 
compost. Dossier CRIQ 640-PE27158 (R1), Rapport final. Recyc-Quebec, $35 \mathrm{p}$.

Deblay S, 2006. Fertilisation et amendements. Educagri éditions, 2 ème édition, Paris, $129 \mathrm{p}$.

FAO et IFA, 2003. Les engrais et leurs applications, IFA, FAO et IMPHOS, 80p

Gala TJ, Camara M, Yao-Kouame A, Keli ZJ, 2011. Gala Bi Rentabilité des engrais minéraux en riziculture pluviale de plateau: Cas de la zone de Gagnoa dans le centre ouest de la Côte d'Ivoire. . J. Appl. Biosci. 46 :3153-3162

Gobat JM, Aragno M, Matthey W, 1998. Le sol vivant. Bases de pédologie - Biologie des sols. Presses polytechniques et universitaires romades, Lausanne, Swisse, 521p.

Kaboré W-TT, Hien E, Zombré P, Coulibaly A, Houot S, Masse D, 2011. Valorisation de substrats organiques divers dans l'agriculture périurbaine de Ouagadougou (Burkina Faso) pour l'amendement et la fertilisation des sols: acteurs et pratiques. Biotechnol. Agron. Soc. Environ. 15(2) : 271-286

Kaho $F$, Yemefack $M$, Feujio-Tegwefouet $P$, Tchanthaouang JC, 2011. Effet combiné de feuilles de Tithonia diversifolia et des engrais inorganiques sur le rendement du maïs et les propriétés d'un sol ferralitique au centre du Cameroun. Tropicultura, 29 (1): 39-45

Kasongo LE, Mwamba MT, Tshipoya MP, Mukalay MJ, Useni SY, Mazinga KM, Nyembo KL, 2013. Réponse de la culture de soja (Glycine max L. (Merril) à l'apport des biomasses vertes de Tithonia diversifolia (Hemsley) A. Gray comme fumure organique sur un Ferralsol à Lubumbashi, R.D. Congo. Journal of Applied Biosciences 63: $4727-4735$

Kotchi V, Yao KA, Sitapha D, 2010. Réponse de cinq variétés de riz à l'apport de phosphate naturel de Tilmesi (Mali) sur les sols acides de la région forestière de Man (Côte d'Ivoire). J. appl. Biosc. 31: 1895-1905.

Maftoun M, Moshiri F, Karimian N, Ronaghi AM, 2004. Effects of two organic wastes in combination withphosphorus on growth and chemical composition ofspinach and soil properties. J. Plant Nutr., 27(9):1635-1651.

Mpundu MM, Useni SY, Mwamba MT, Kateta MG, Mwansa M, llunga K, Kamengwa KC, Kyungu K, Nyembo KL, 2013. Teneurs en éléments traces métalliques dans les sols de différents jardins potagers de la ville minière de
Lubumbashi et risques de contamination des cultures potagères. Journal of Applied Biosciences 65:4957 - 4968

Mulaji KC, 2011. Utilisation des composts de biodéchets ménagers pour l'amélioration de la fertilité des sols acides de la province de Kinshasa (République Démocratique du Congo). Thèse de doctorat, université de Liège- Gembloux Agro-Biotech, 220p.

Munyemba KF, 2010. Quantification et modélisation de la dynamique paysagère dans la région de Lubumbashi : évaluation de limpact écologique des dépositions issues de la pyrométallurgie. Thèse de doctorat, Université de Lubumbashi, 284p

Munyemba KF, Bamba I, Kabulu DJ, Amisi M, Veroustrate F, Ngongo LM, Bogaert J, 2008. Occupation des sols dans le cône de pollution à Lubumbashi. Ann. Fac. Sc. Agro., 1(2): 1925

N'Dayegamiye A, Drapeau A, 2009. Influence de la nature des fertilisants apportés sur la dynamique de la structure et les teneurs en carbone et en substances humiques pour un loam argileux Sainte-Rosalie. Agrosol. 20 (1) : 15-22.

N'Dayegamiye A, Drapeau A, Laverdière MR, 2005. Effets des apports de composts de résidus ménagers sur les rendements des cultures et certaines propriétés du sol. Agrosol. 16 (2) $: 57-71$.

N'Dayegamiye A, Drapeau A, Huard S, Thibault Y, 2004. Intégration de boues mixtes et de fumiers dans des rotations agricoles : réponse des cultures et interactions avec les propriétés du sol. Agrosol. 15 (2) : 83-90.

N'Dienor M, 2006. Fertilité et gestion de la fertilisation dans les systèmes maraîchers périurbains des pays en développement : intérêts et limites de la valorisation agricole des déchets urbains dans ces systèmes, cas de l'agglomération d'Antananarivo (Madagascar). Thèse de doctorat, Université d'Antanarivo, Ecole Supérieure des Sciences Agronomiques, 242p

Nyembo KL, Useni SY, Chukiyabo KM, Tshomba KJ, Ntumba NF, Muyambo ME, Kapalanga KP, Mpundu MM, Bugeme MD, Baboy LL, 2013. Rentabilité économique du fractionnement des engrais azotés en culture de maïs (Zea mays L.): cas de la ville de Lubumbashi, sud-est de 
la RD Congo. Journal of Applied Biosciences 65:4945- 4956

Nyembo KL, Useni SY, Mpundu MM, Bugeme MD, Kasongo LE, Baboy LL, 2012. Effets des apports des doses variées de fertilisants inorganiques (NPKS et Urée) sur le rendement et la rentabilité économique de nouvelles variétés de Zea mays L. à Lubumbashi, SudEst de la RD Congo. Journal of Applied Biosciences 59: 4286- 4296

Pypers $\mathrm{P}, 2010$. Integrated soil fertility management: The TSBF definitions, seminar at UEA, 11th May 2010. Bukavu, DRC

Sanginga N, Woomer $P, 2009$. Integrated soil fertility management in Africa: principles, practices and process developpement, TSBF-CIAT and FORMAT, Nairobi Kenya,

Tejada M, Hernandez MT,Garcia C, 2006. Application of two organic amendments on soil restoration: Effects on the soil biological properties. J Environ. Qual., 35: 1010-1017.

Useni SY, Chukiyabo KM, Tshomba KJ, Muyambo ME, Kapalanga KP, Ntumba NF, Kasangij A-KP, Kyungu K, Baboy LL, Nyembo KL, Mpundu MM, 2013. Utilisation des déchets humains recyclés pour l'augmentation de la production du maïs (Zea mays L.) sur un ferralsol du sudest de la RD Congo. Journal of Applied Biosciences 66:5070 - 5081

Useni SY, Baboy LL, Nyembo KL, Mpundu MM, 2012. Effets des apports combinés de biodéchets et de fertilisants inorganiques sur le rendement de trois varieties de Zea mays L. cultivées dans la région de Lubumbashi. Journal of Applied Biosciences 54: 3935- 3943

Vanlauwe B, Tittonell P, Mukalama J, 2006. Withinfarm soil fertility gradients affect response of maize to fertilizer application in western Kenya. Nutrient Cycling Agroecosystem 76:171-182 